Supplementary Information

\title{
Toxaphene Deposition to Lake Ontario via \\ Precipitation, 1994-1998
}

Deborah A. Burniston*, William M. J. Strachan, Robert J. Wilkinson

Supplementary information has seven pages including the cover and contains two tables of data. 
Table 1: Homolog and Total Toxaphene Concentrations in Point Petre Precipitation $(1994,1995,1996,1997,1998)$

\begin{tabular}{|c|c|c|c|c|c|c|c|}
\hline \multirow{2}{*}{$\begin{array}{c}\text { Mid-Period } \\
\text { Julian Day }\end{array}$} & \multirow{2}{*}{$\begin{array}{c}\text { No. of } \\
\text { Samples }\end{array}$} & \multicolumn{4}{|c|}{ 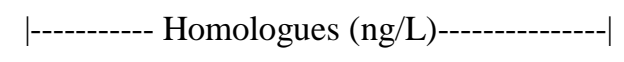 } & \multirow{2}{*}{$\begin{array}{c}\text { Total } \\
\text { Toxaphene }\end{array}$} & \multirow[t]{2}{*}{ Season $^{\mathrm{a}}$} \\
\hline & & Hexa- & Hept- & Octa- & Nona- & & \\
\hline \multicolumn{8}{|l|}{1994} \\
\hline 204 & 3 & 0.070 & 0.70 & 0.54 & 0.050 & 1.4 & Summer \\
\hline 330 & 3 & 0.011 & 0.44 & 0.52 & 0.060 & 1.0 & Autumn \\
\hline \multicolumn{8}{|l|}{1995} \\
\hline 5 & 2 & 0.060 & 0.50 & 0.28 & 0.090 & 0.93 & Winter \\
\hline 32 & 4 & 0.0040 & 0.15 & 0.092 & 0.011 & 0.26 & \\
\hline 72 & 6 & 0.010 & 0.45 & 0.61 & 0.11 & 1.2 & Spring \\
\hline 115 & 3 & 0.047 & 0.71 & 1.0 & 0.16 & 1.9 & \\
\hline 143 & 3 & 0.054 & 0.31 & 0.23 & 0.041 & 0.64 & Summer \\
\hline 227 & 3 & 0.0095 & 0.26 & 0.57 & 0.022 & 0.86 & \\
\hline
\end{tabular}




\begin{tabular}{|c|c|c|c|c|c|c|c|}
\hline 255 & 3 & 0.010 & 0.26 & 0.32 & 0.035 & 0.63 & \multirow[t]{3}{*}{ Autumn } \\
\hline 283 & 3 & 0.0042 & 0.19 & 0.22 & 0.033 & 0.44 & \\
\hline 311 & 3 & 0.0080 & 0.19 & 0.22 & 0.025 & 0.44 & \\
\hline 338 & 3 & 0.0010 & 0.075 & 0.031 & $<0.009$ & 0.12 & Winter ('96) \\
\hline \multicolumn{8}{|l|}{1996} \\
\hline 16 & 4 & 0.014 & 0.45 & 0.51 & 0.11 & 1.1 & \multirow[t]{2}{*}{ Winter } \\
\hline 58 & 3 & 0.010 & 0.46 & 0.54 & 0.090 & 1.1 & \\
\hline 99 & 4 & 0.022 & 0.81 & 0.84 & 0.21 & 1.9 & \multirow{2}{*}{ Spring } \\
\hline 142 & 3 & 0.030 & 0.46 & 0.47 & 0.10 & 1.1 & \\
\hline 170 & 3 & 0.088 & 0.44 & 0.48 & 0.080 & 1.1 & \multirow[t]{3}{*}{ Summer } \\
\hline 198 & 3 & 0.040 & 0.30 & 0.26 & 0.050 & 0.65 & \\
\hline 226 & 3 & 0.025 & 0.36 & 0.39 & 0.070 & 0.85 & \\
\hline 254 & 3 & 0.0060 & 0.11 & 0.11 & 0.020 & 0.25 & \multirow[t]{3}{*}{ Autumn } \\
\hline 282 & 3 & 0.0060 & 0.18 & 0.19 & 0.040 & 0.42 & \\
\hline 310 & 3 & 0.0020 & 0.23 & 0.25 & 0.040 & 0.52 & \\
\hline 1997 & & & & & & & \\
\hline 338 & 3 & 0.030 & 0.30 & 0.32 & 0.030 & 0.68 & Winter \\
\hline
\end{tabular}




\begin{tabular}{|c|c|c|c|c|c|c|c|}
\hline 1 & 3 & 0.0046 & 0.28 & 0.40 & 0.024 & 0.71 & \\
\hline 28 & 3 & 0.034 & 0.37 & 0.60 & 0.069 & 1.1 & \\
\hline 56 & 3 & 0.023 & 0.33 & 0.42 & 0.045 & 0.81 & \\
\hline 84 & 3 & 0.023 & 0.40 & 0.53 & 0.063 & 1.0 & Spring \\
\hline 112 & 3 & 0.023 & 0.41 & 0.67 & 0.11 & 1.2 & \\
\hline 140 & 3 & 0.15 & 0.19 & 0.27 & 0.078 & 0.68 & \\
\hline 196 & 3 & 0.0030 & 0.034 & 0.055 & $<0.01$ & 0.089 & Summer \\
\hline 224 & 3 & 0.0063 & 0.11 & 0.19 & 0.026 & 0.33 & \\
\hline 252 & 3 & 0.014 & 0.10 & 0.25 & 0.038 & 0.40 & Autumn \\
\hline 280 & 3 & 0.037 & 0.19 & 0.22 & 0.044 & 0.49 & \\
\hline 308 & 3 & 0.010 & 0.26 & 0.30 & 0.037 & 0.61 & \\
\hline 1998 & & & & & & & \\
\hline 336 & 3 & 0.024 & 0.43 & 0.50 & 0.14 & 1.1 & Winter \\
\hline 364 & 3 & 0.016 & 0.24 & 0.33 & 0.040 & 0.62 & \\
\hline 27 & 3 & 0.14 & 3.3 & 4.9 & 0.41 & 8.7 & \\
\hline 55 & 3 & 0.0030 & 0.16 & 0.24 & 0.22 & 0.42 & \\
\hline 83 & 3 & 0.021 & 0.31 & 0.69 & 0.054 & 1.1 & \\
\hline & & & & & & & \\
\hline
\end{tabular}




\begin{tabular}{|r|c|c|c|c|c|c|c|}
\hline 111 & 3 & 0.028 & 0.16 & 0.17 & 0.031 & 0.39 & \multirow{2}{*}{ Spring } \\
\hline 139 & 3 & 0.070 & 0.92 & 1.7 & 0.23 & 2.9 & Summer \\
\hline 167 & 3 & 0.013 & 0.038 & 0.12 & 0.017 & 0.19 & \\
\hline 223 & 3 & 0.082 & 0.36 & 0.72 & 0.91 & 1.3 & \\
\hline 251 & 3 & 0.0020 & 0.053 & 0.025 & $<0.009$ & 0.079 & \multirow{2}{*}{ Autumn } \\
\hline 279 & 3 & 0.0070 & 0.099 & 0.13 & 0.016 & 0.25 & \\
\hline 307 & 3 & 0.011 & 0.21 & 0.25 & 0.029 & 0.50 & \\
\hline
\end{tabular}

a The IADN convention for the seasons is followed in this paper with the first period -winter 1995 -- being made up of samples only from January and February.

Table 2: Seasonal and Annual Concentrations and Flux Rates of Toxaphene at Point

Petre $(1995,1996,1997,1998)$

\begin{tabular}{|c|c|c|c|c|c|c|}
\hline Season & Hexa- & Hept- & Octa- & Nona- & Total & Precip.(mm) \\
\hline |---'-- & --------- & -------- & $-1995--$ & ---------- & . & -1 \\
\hline \multicolumn{7}{|c|}{ Vol.Wtd.Concentration ng/L (Flux Rate $\mathrm{ng} / \mathrm{m}^{2} /$ period) } \\
\hline Winter & $0.029(5.7)$ & $0.30(59)$ & $0.18(36)$ & $0.053(10)$ & $0.56(110)$ & 198 \\
\hline Spring & $0.027(3.6)$ & $0.57(77)$ & $0.80(110)$ & $0.13(18)$ & $1.5(200)$ & 135 \\
\hline
\end{tabular}




\begin{tabular}{|c|c|c|c|c|c|c|}
\hline Summer & $0.035(6.5)$ & $0.29(54)$ & $0.37(68)$ & $0.032(6.0)$ & $0.73(140)$ & 186 \\
\hline Autumn & $0.0071(2.6)$ & $0.21(76)$ & $0.25(91)$ & $0.032(12)$ & $0.50(180)$ & 364 \\
\hline Annual & $0.020(18)$ & $0.30(260)$ & $0.34(300)$ & $0.053(47)$ & $0.71(630)$ & 883 \\
\hline \multicolumn{7}{|c|}{ Vol.Wtd.Concentration ng/L (Flux Rate $\mathrm{ng} / \mathrm{m}^{2} /$ period) } \\
\hline Winter & $0.010(2.1)$ & $0.37(77)$ & $0.42(87)$ & $0.082(17)$ & $0.88(180)$ & 207 \\
\hline Spring & $0.026(6.0)$ & $0.64(150)$ & $0.66(150)$ & $0.16(37)$ & $1.5(340)$ & 229 \\
\hline Summer & $0.052(13)$ & $0.37(95)$ & $0.38(97)$ & $0.070(18)$ & $0.87(220)$ & 256 \\
\hline Autumn & $0.0050(1.7)$ & $0.17(58)$ & $0.17(58)$ & $0.030(10)$ & $0.38(130)$ & 339 \\
\hline Annual & $0.021(22)$ & $0.37(380)$ & $0.38(390)$ & $0.080(82)$ & $0.85(880)$ & 1031 \\
\hline Season & Hexa- & Hept- & Octa- & Nona- & Total & Precip.(mm) \\
\hline Vol.Wtd.Concen & ation ng/L (F & Rate $\mathrm{ng} / \mathrm{m}^{2}$ & riod) & & & \\
\hline Winter & $0.021(5.6)$ & $0.33(88)$ & 0.48 (130) & $0.047(13)$ & $0.88(240)$ & 268 \\
\hline
\end{tabular}




\begin{tabular}{|c|c|c|c|c|c|c|}
\hline Spring & $0.047(10)$ & $0.36(77)$ & $0.53(110)$ & $0.086(18)$ & $1.0(210)$ & 213 \\
\hline Summer & $0.0055(1.1)$ & $0.092(19)$ & 0.15 & $0.020(4.2)$ & $0.27(56)$ & 208 \\
\hline Autumn & $0.019(5.1)$ & $0.20(54)$ & $0.26(70)$ & $0.039(10)$ & $0.52(140)$ & 268 \\
\hline Annual & $0.023(22)$ & $0.25(240)$ & $0.36(340)$ & $0.048(46)$ & $0.68(650)$ & 957 \\
\hline \multicolumn{7}{|c|}{ | } \\
\hline \multicolumn{7}{|c|}{ Vol.Wtd.Concentration ng/L (Flux Rate $\mathrm{ng} / \mathrm{m}^{2} /$ period) } \\
\hline Winter & $0.015(3.7)$ & $0.33(82)$ & $0.47(120)$ & $0.060(15)$ & $0.88(220)$ & 248 \\
\hline Spring & $0.037(7.4)$ & $0.43(86)$ & $0.80(160)$ & $0.10(20)$ & $1.4(280)$ & 199 \\
\hline Summer & $0.042(12)$ & $0.17(49)$ & $0.37(110)$ & $0.047(13)$ & $0.63(180)$ & 287 \\
\hline Autumn & $\begin{array}{c}0.0060 \\
(0.95)\end{array}$ & $0.11(17)$ & $0.12(19)$ & $0.013(2.1)$ & 0.25 & 158 \\
\hline Annual & $0.028(25)$ & $0.25(220)$ & $0.44(390)$ & $0.054(48)$ & 0.77 (690) & 892 \\
\hline
\end{tabular}

